\title{
Precision CMB measurements with long-duration stratospheric balloons: activities in the Arctic
}

\author{
P. de Bernardis ${ }^{1}$, S. Masi ${ }^{1}$ \\ for the OLIMPO and LSPE teams \\ ${ }^{1}$ Dipartimento di Fisica, Sapienza Universitá di Roma, P.le A. Moro 200185 Roma, Italy \\ email: paolo.debernardis@roma1.infn.it
}

\begin{abstract}
We report on the activities preparing long duration stratospheric flights, suitable for CMB (Cosmic Microwave Background) measurements, in the Arctic region. We focus on pathfinder flights, and on two forthcoming experiments to be flown from Longyearbyen (Svalbard islands): the OLIMPO Sunyaev-Zeldovich spectrometer, and the Large-Scale Polarization Explorer (LSPE).
\end{abstract}

Keywords. Cosmic Microwave Background, Arctic, Stratospheric Balloons

\section{Introduction}

In 1998 the BOOMERanG experiment started the use of long-duration balloon (LDB) flights in the Antarctic stratosphere to perform precision Cosmic Microwave Background (CMB) anisotropy measurements, producing detailed maps of the CMB where causal horizons in the cosmic photosphere are fully resolved (B98, see e.g. de Bernardis et al. 2000). This was followed by the successful Antarctic flight of BOOMERanG-polarization (B03, see e.g. Montroy et al. 2006). Nowadays the EBEX experiment (see ReichbornKjennerud et al. 2010) is going to be flown on a similar long duration flight, promising unprecedented sensitivity in the measurement of CMB polarization, as well as the SPIDER experiment (see Filippini et al. 2010), targeting at larger angular scales.

The Arctic region can be used to perform similar LDB flights. Developing these flights will double the availability of long-duration flight opportunities every year, reducing the waiting time for users, and will enable access to the northern hemisphere, which cannot be observed during Antarctic flights. Longyearbyen, in the Svalbard islands, is at a latitude of $78^{\circ} \mathrm{N}$ (roughly at the antipodes of McMurdo) and can provide all the required infrastructure. A number of small-payload flights and one heavy-lift payload have been successfully launched from the Longyearbyen airport, demonstrating the feasibility of long duration flights in the Arctic (Peterzen et al. 2008). In addition, we have launched small payloads from the Dirigibile Italia station in Ny-Alesund (also in the Svalbard islands) during the Arctic winter, demonstrating the eastward trajectory (see below). In the following we shortly report on the Arctic flights, and then focus on two CMB payloads we plan to launch from Longyearbyen: OLIMPO and LSPE.

\section{Long Duration Flights in the Arctic}

The first LDB flight fully circumnavigating the north pole at high latitudes was launched from Longyearbyen in the summer of 2006, by an international team composed of personnel from the Italian Space Agency, Andoya Rocket Range, Sapienza Universitá 


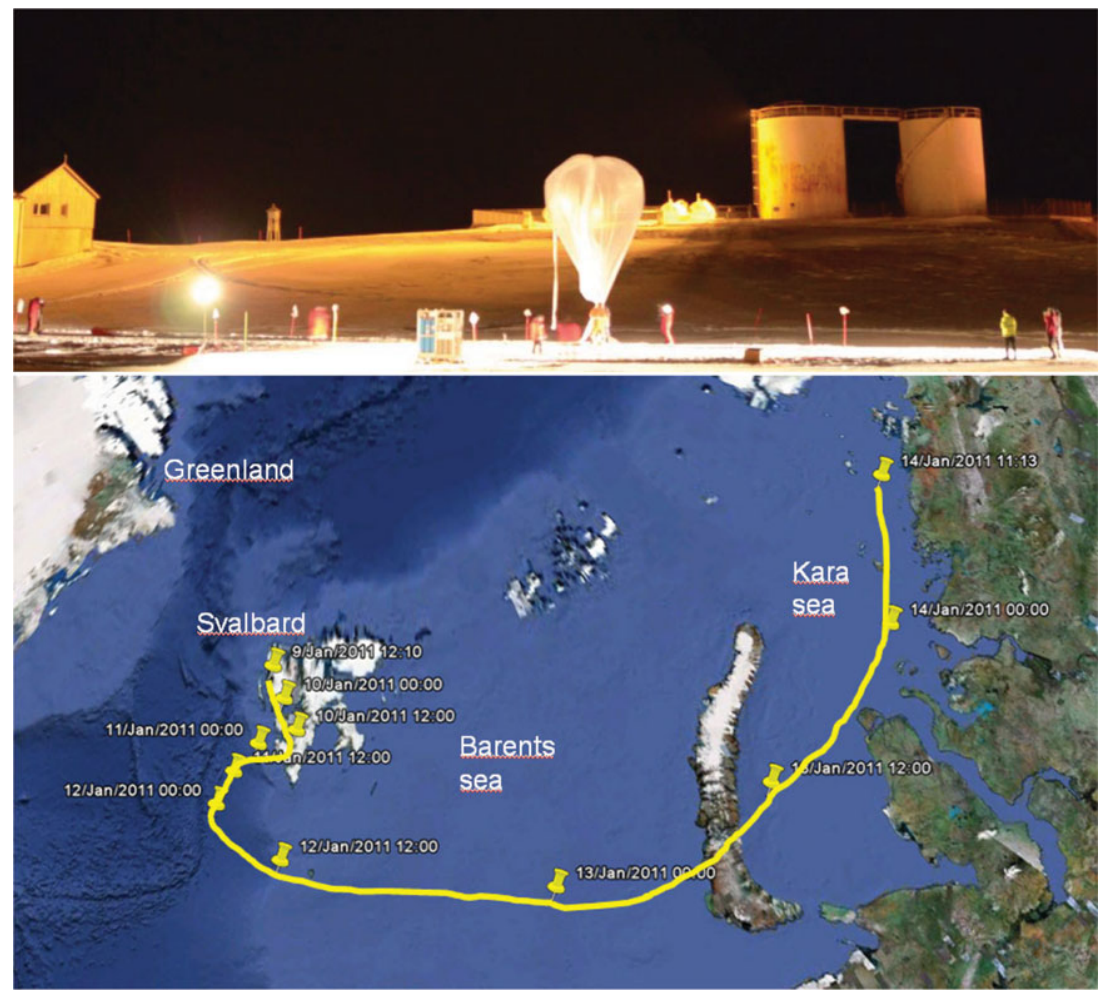

Figure 1. Top: Launch of a path-finder balloon from Ny-Alesund (Svalbard, Jan. 2012); bottom: Ground-track of a path-finder balloon launched from Ny-Alesund (Svalbard, Jan. 2011)

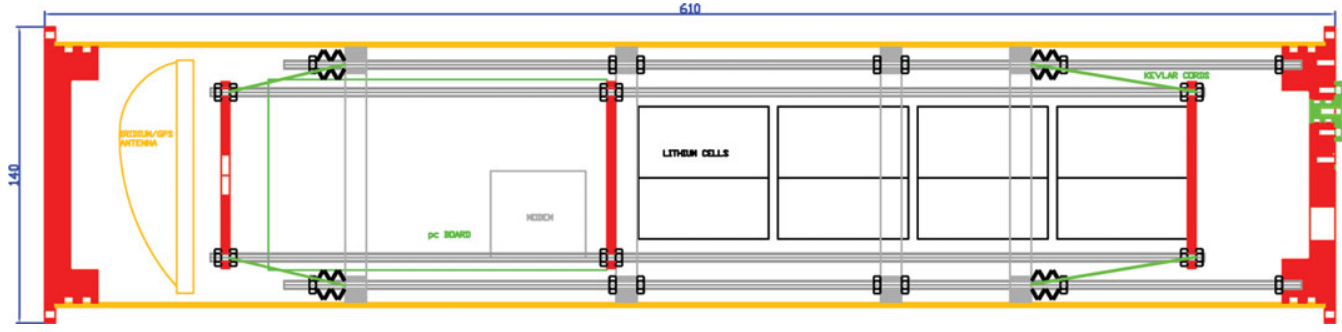

Figure 2. Sketch of the light-weight payload used to study stratospheric circulation in the Arctic during the polar winter. Dimensions in $\mathrm{mm}$.

di Roma, Istituto Nazionale di Geofisica, ISTAR. The ground track of that flight can be found in Peterzen et al. 2008. In 2009 the same team launched the heavy-lift $(>1$ ton) SORA experiment, with a $800,000 \mathrm{~m}^{3}$ balloon, also from Longyearbyen. The ground track for that flight is shown in Flamini \& Pirrotta 2011.

A team composed of personnel from La Sapienza, ISTAR and CNR, launched in Jan. 2011 and Jan. 2012 path-finder balloons $\left(3,000\right.$ and 9,000 $\left.\mathrm{m}^{3}\right)$ from the Dirigibile Italia base in Ny-Alesund (Svalbard), to demonstrate the east-bound trajectories during the polar winter. The ground track of one of these flights is shown in Fig. 1.

The balloon lifted a lightweight $(5 \mathrm{~kg})$ payload consisting of a GPS receiver, temperature and pressure sensors, an Iridium SBD communication system, a radiation-hard 

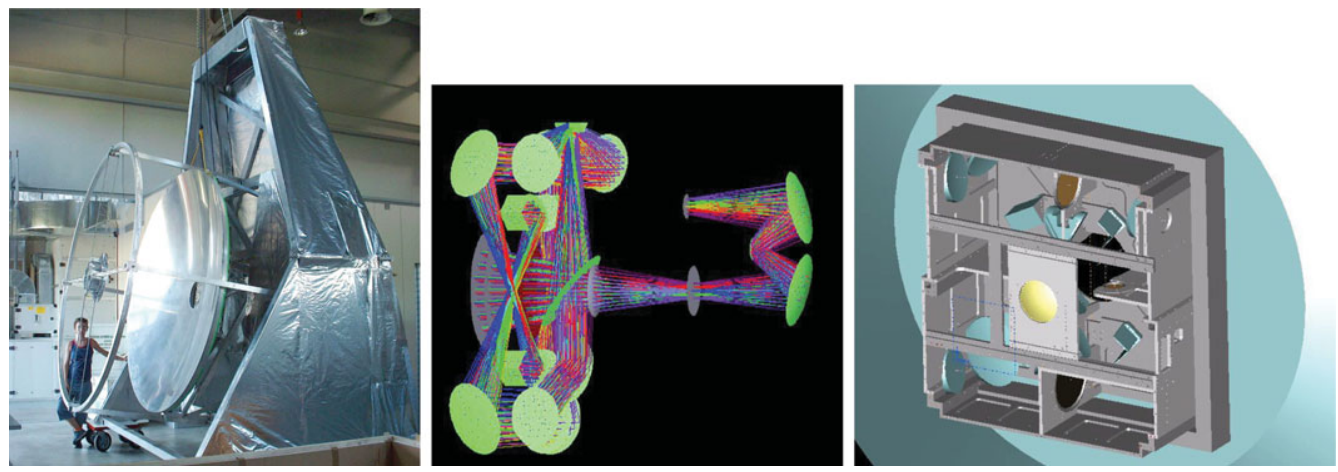

Figure 3. Left: The OLIMPO payload with solar panels, ground-shields and sun-shields removed. Center: Optical configuration of the lossless Martin-Pupplett DFTS for OLIMPO (on the left in the figure) and existing reimaging optics inside the cryostat (on the right in the figure); Right: Rendering of the mechanical setup for the DFTS: the center lens and the following wedge can be shifted on command, allowing the beam to by-pass the interferometer, for photometric measurements.

microcontroller and a battery pack. The main challenge in the preparation of a payload for a long duration flight in the polar night is thermal insulation. The system has to operate in an environment with a pressure of a few mbar and a temperature of $\sim-80^{\circ} \mathrm{C}$, supplied only by batteries. In order to minimize the weight of the system, we used lithium cells, which maximize the stored energy to weight ratio, and insulated very carefully the battery pack and the system electronics to avoid the use of electrical heaters. The system is surrounded by mylar superinsulation, suspended by kevlar cords, and inserted in a vacuum vessel to minimize heat dispersion. A sketch of the payload is shown in Fig. 2. The system has been able to operate in the harsh conditions of the polar winter stratosphere with just $1 \mathrm{~W}$ of power.

Although a full winter circumnavigation has not been achieved yet, the east-bound trajectory has been demonstrated. We plan to repeat the path-finders launch campaign in the coming winter seasons, to improve the statistics and to find the best launch period during the winter.

\section{OLIMPO}

The OLIMPO (Masi et al. 2008) balloon payload is a $2.6 \mathrm{~m}$ millimeter-wave telescope aimed at measuring the Sunyaev-Zeldovich (SZ, Sunyaev \& Zeldovich 1972) effect in clusters of galaxies. It works at high frequencies (up to $500 \mathrm{GHz}$ ), which cannot be observed from the ground, and with angular resolution higher than the recent Planck survey (Planck Collaboration, 2011a). At $\sim 450 \mathrm{GHz}$ (near the positive maximum of the SZ spectral brightness), the angular resolution of OLIMPO is comparable to the resolution of the $10 \mathrm{~m}$ class mm-wave telescopes operating at $\sim 140 \mathrm{GHz}$ in Antarctica (see Carlstrom et al. 2011) and in Atacama (see Swetz et al. 2011 and Schwan et al. 2011). The OLIMPO payload has been recently upgraded with a lossless, differential, low-resolution $(6 \mathrm{GHz})$, Fourier transform spectrometer (DFTS), which was placed as a plug-in between the rear part of the primary mirror and the window of the cryostat (see Fig. 3).

This instrument can measure 33 independent frequency bands, located below, across and above the null of the SZ spectrum (see Table 1). The sensitivity (NEPD, noise equivalent power density) has been computed per detector, per beam, and assuming photon 
Table 1. Main features of the OLIMPO spectroscopic instrument

\begin{tabular}{|c|c|c|c|c|c|}
\hline $\begin{array}{c}\text { Band } \\
(\mathrm{GHz})\end{array}$ & Sub-bands & $\begin{array}{c}\text { FWHM } \\
\left({ }^{\prime}\right)\end{array}$ & $\begin{array}{c}\text { Array } \\
\text { Elements }\end{array}$ & $\begin{array}{c}\text { Background } \\
(\mathrm{pW})\end{array}$ & $\begin{array}{c}\text { NEPD } \\
(\mathrm{fW} \sqrt{s} / G H z)\end{array}$ \\
\hline $135-160$ & 4 & 4.0 & 19 & 6 & 5 \\
$180-250$ & 12 & 3.0 & 19 & 20 & 12 \\
$330-365$ & 6 & 1.7 & 23 & 7 & 9 \\
$450-515$ & 11 & 1.2 & 23 & 14 & 14 \\
\hline
\end{tabular}

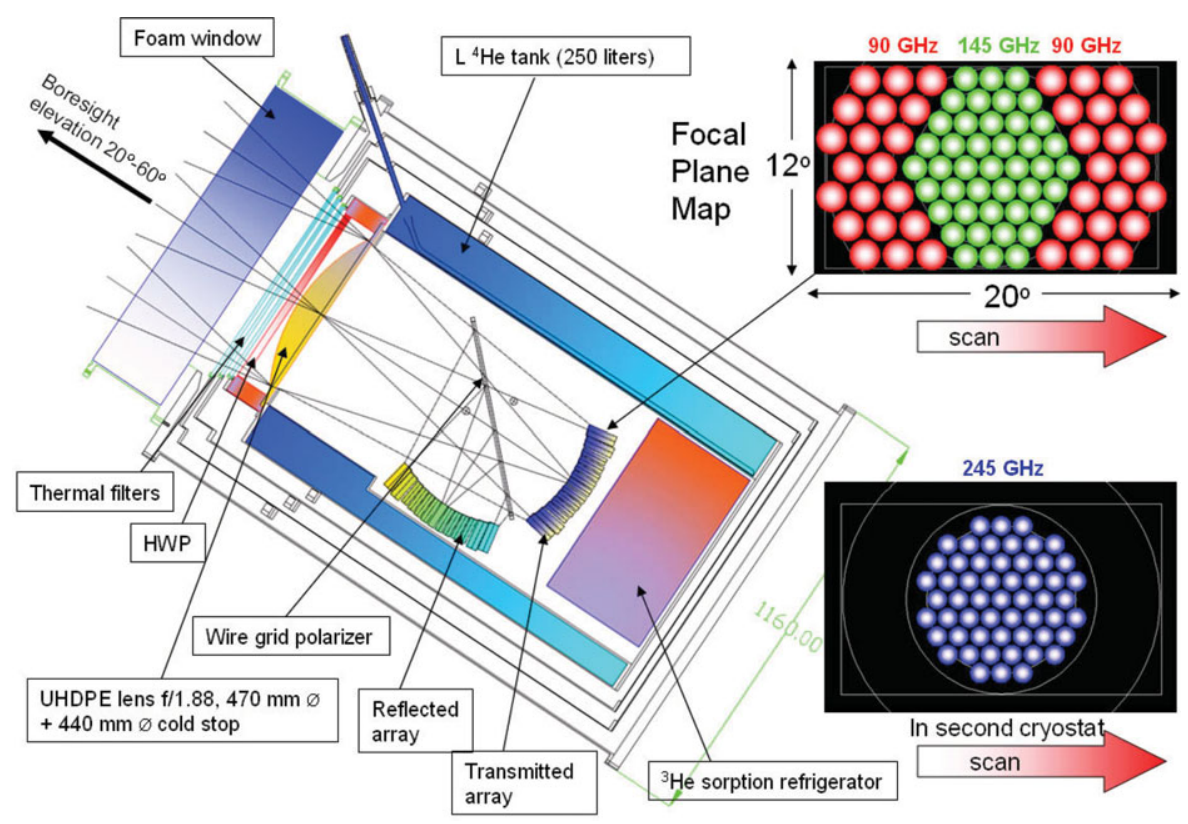

Figure 4. Left: The SWIPE instrument on the LSPE.

noise limited detectors. The DFTS is intrinsically rejecting the large common mode signal from the telescope, the residual atmosphere and the CMB monopole. The effectiveness of this instrument in removing degeneracies between cluster (and foreground) parameters has been discussed in de Bernardis et al. 2012. OLIMPO will carry-out an ambitious observation program, including spectroscopic observations of the SZ in 40 known clusters, plus a $10^{\circ} \times 10^{\circ}$ blind deep-sky survey. In addition, OLIMPO represents a first step in the development of space-based differential spectrometers for the $\mathrm{mm}$ and sub-mm bands, which will eventually lead to a DFTS instrument for the $10 \mathrm{~m}$ space telescope on the Millimetron mission. The flight of OLIMPO is scheduled for June 2013.

\section{LSPE}

The Large-Scale Polarization Explorer (see LSPE collaboration 2012) is a balloonborne mission polarimeter aimed at measuring the polarization of the CMB at large angular scales, to constrain the curl component of CMB polarization (B-modes) produced by cosmic inflation. The target is to constrain the ratio of tensor to scalar perturbations amplitudes down to $r=0.03$, at $99.7 \%$ confidence. This will be achieved measuring multi-color wide maps of the mm sky, in order to beat cosmic variance and to monitor the polarized foreground generated in our Galaxy by synchrotron emission and interstellar dust emission. The mission is optimized for large angular scales, with coarse angular 
Table 2. Main features of the LSPE polarimeters

\begin{tabular}{|c|c|c|c|}
\hline $\begin{array}{c}\text { Band } \\
(\mathrm{GHz})\end{array}$ & $\begin{array}{c}\text { FWHM } \\
(\mathrm{deg})\end{array}$ & $\begin{array}{c}\text { Array } \\
\text { Elements }\end{array}$ & $\begin{array}{c}\mathbf{N E T}_{\text {array }} \\
(\mu K \sqrt{s})\end{array}$ \\
\hline 43 & 1.0 & 49 & 33 \\
90 & 0.5 & 7 & 104 \\
\hline 95 & 1.8 & 80 & 1.9 \\
145 & 1.5 & 86 & 1.8 \\
245 & 1.2 & 110 & 1.9 \\
\hline
\end{tabular}

resolution (around $1.5^{\circ}$ degrees FWHM), and wide sky coverage (25\% of the sky). To achieve this, the payload will fly on a circumpolar LDB in the polar winter. Using the Earth as a giant solar shield, the instrument will spin in azimuth, observing a large fraction $(>25 \%)$ of the northern sky. The payload will host two instruments. STRIP (the STRatospheric Italian Polarimeter), an array of coherent polarimeters using cryogenic HEMT amplifiers, will survey the sky at 43 and $90 \mathrm{GHz}$ (see Bersanelli et al. 2012). SWIPE (the Short Wavelength Instrument for the Polarization Explorer), an array of bolometric polarimeters, will survey the same sky region in three bands at 95, 145 and $245 \mathrm{GHz}$ (see de Bernardis et al. 2012b). The resulting wide frequency coverage will allow optimal control of the polarized foregrounds, with comparable angular resolution at all frequencies. While the main purpose of STRIP is to monitor low-frequency polarized foregrounds, SWIPE provides high sensitivity by means of arrays of large-throughput bolometers. In fact, each bolometer of SWIPE collects $>20$ modes of the incoming radiation, boosting by a factor $>4$ the sensitivity with respect to single-mode detectors. Polarization modulation is achieved by means of a rotating half-wave-plate and a static polarizer in front of the bolometers. The main characteristics of LSPE are summarized in Table 2. In Fig. 4 we show the setup of the SWIPE polarimeters.

The flight of LSPE is scheduled for January 2015.

Acknowledgements: The OLIMPO and LSPE projects have been funded by the Italian Space Agency. The balloon campaigns at Ny-Alesund have been supported by Programma Nazionale Ricerche in Antartide - CNR contract 2010-A3.02. We gratefully acknowledge the support of the CNR team at the Dirigibile Italia base in Ny-Alesund. The OLIMPO collaboration includes teams from University of Rome La Sapienza, IFACCNR Firenze, INGV Roma, University of Milano Bicocca, Dept. of Physics and Astron. Univ. of Cardiff, NIST Boulder, CEA Saclay, INPE Brasil, Agenzia Spaziale Italiana. See Masi et al. 2008 and de Bernardis et al. 2012 for the full list of collaborators. The LSPE collaboration includes teams from University of Rome La Sapienza, University of Milano, University of Milano Bicocca, IFAC-CNR Firenze, IASF-INAF Bologna, University of Firenze, Cavendish Laboratory Cambridge, University of Trieste, Jodrell Bank CFA, University of Manchester, IEIIT-CNR Torino, Istituto Nazionale di Geofisica e Vulcanologia Roma, IASF-INAF Milano, Agenzia Spaziale Italiana, OAT-INAF Trieste. See LSPE collaboration 2012 for the full list of collaborators.

\section{References}

Bersanelli, M., et al., 2012, in Proceedings of the SPIE Astronomical Telescopes + Instrumentation 2012 Conference - Ground-based and Airborne Instrumentation for Astronomy IV, astro-ph/1208.0164

Carlstrom, J. E., et al., 2011 Publ. Astron. Soc. of the Pacific 123, 568

de Bernardis, P., et al., 2000, Nature, 404, 955

de Bernardis, P., et al., 2012, Astronomy \& Astrophysics, 538, A86 
de Bernardis, P., et al., 2012b, in Proceedings of the SPIE Astronomical Telescopes + Instrumentation 2012 Conference - Ground-based and Airborne Instrumentation for Astronomy $I V$, astro-ph/1208.0282

Filippini, J.P., et al., in: Proceedings for SPIE Millimeter, Submillimeter, and Far-Infrared Detectors and Instrumentation for Astronomy $V$ astro-ph/1106.2158

Flamini, E. \& Pirrotta, S., 2011, Mem. Soc. Astron. It. Supp. 16, 145

LSPE Collaboration, Amico G. et al., 2012, Proceedings of the SPIE Astronomical Telescopes + Instrumentation 2012 Conference - Ground-based and Airborne Instrumentation for Astronomy $I V$, astro-ph/1208.0281

Masi, S., et al., 2008, Mem. Soc. Astron. It. Supp., 79, 887

Montroy, T., et al., 2006, Ap.J., 647, 813

Peterzen, S., et al. 2008, Mem. Soc. Astron. It. 79, 792

Planck Collaboration, Ade, P. A. R., Aghanim, N., \& Arnaud, M. e. a., 2011a, Astronomy \&6 Astrophysics 536, A8

Reichborn-Kjennerud, B., et al. in: Proceedings for SPIE Millimeter, Submillimeter, and FarInfrared Detectors and Instrumentation for Astronomy $V$ astro-ph/1007.3672

Schwan, D., et al. 2011, Review of Scientific Instruments 82, 091301

Sunyaev, R. A. and Zeldovich, Y. B., 1972, Comments on Astrophysics and Space Physics 4, 173

Swetz, D. S., et al., 2011, Ap.J.Suppl., 194, 41 